\title{
Optimal Guaranteed Cost Control of Discrete-Time Linear Systems subject to Structured Uncertainties
}

\author{
Carlos M. Massera ${ }^{\mathrm{a}}$, Marco H. Terra ${ }^{\mathrm{b}}$ and Denis F. Wolf ${ }^{\mathrm{a}}$ \\ ${ }^{a}$ Institute of Mathematics and Computer Science, University of São Paulo, São Carlos, \\ Brazil; bSão Carlos School of Engineering, University of São Paulo, São Carlos, Brazil
}

\begin{abstract}
In this paper, we propose state- and static output-feedback generalized guaranteed cost control (GCC) approaches for discrete-time linear systems subject to normbounded structured parametric uncertainties. This method enables the convex synthesis for a more general class of systems, where uncertainties are uncorrelated block diagonal, and no feed-through uncertainty is multiplicative with control input ones. It also provides necessary and sufficient conditions for state-feedback and sufficient conditions for static output-feedback. We also present a comparative study among the proposed controllers, standard Linear Quadratic Regulator, and Guaranteed Cost Controller found in the literature.
\end{abstract}

\section{Introduction}

The Linear Quadratic Regulator (LQR) (Kalman et al. 1960) is an optimal control approach which aims to drive states of a linear system to its origin through the minimization of a quadratic cost functional. Safonov and Athans (Safonov and Athans 1976) have shown that such controller has $6 d B$ gain margin and $60^{\circ}$ phase margin if the system dynamics is assumed to be known. However, when it is subject to uncertainties, optimal closed-loop performance cannot be obtained since robustness properties are no longer guaranteed (Douglas 1991).

To address this problem, several authors in the last decades have proposed different approaches to solve it. Petersen (Petersen 1987), for instance, proposed a Guaranteed Cost Controller (GCC) for a continuous-time linear system subject to $l 2$-normbounded uncertainties based on the solution of Algebraic Riccati Equations (ARE). Such method synthesizes a controller which presents robust asymptotic stability and provides a guaranteed upper bound to a quadratic cost function used as performance measurement. Xie and Soh (Xie and Soh 1993) applied such approach to discrete-time systems, which Petersen et al. (Petersen et al. 1998) extended to a more general class of systems through the use of Linear Matrix Inequality (LMI). Previous studies applied this class of synthesis to several types of systems and controllers, including static and dynamic output feedback controllers. Some examples can be seen in: (Garcia et al. 2003, Moheimani and Petersen 1996, Yu and Chu 1999, Chen et al. 2003, Boukas and Shi 1998, Yang and Zhao 2016, Qiu et al. |2016, Wang et al. |2016).

A challenging aspect of most robust synthesis problems is its inherent non-convexity, even when no uncertainties are considered. This topic has attracted significant research

Corresponding author: Carlos M. Masssera. Email: carlos.magalhaes.filho@usp.br 
interest in the past two decades. Most of the approaches proposed for such class of problems are based on dilated variables, first proposed by Oliveira et al. (de Oliveira et al. 1999a) for discrete time systems and later extended by Apkarian et al. (Apkarian et al. |2001) for continuous time systems. Some examples of applications of such method are: Output feedback synthesis (i.e., (Lee et al. 2006), (Xie 1996), (Dong and Yang 2007), (Peaucelle and Ebihara 2014), (Arzelier et al.|2003)); systems subject to delays (i.e., (He et al.|2004), (Li et al.||2016)); systems subject to Markovian jumps (i.e. (de Souza 2003), (Morais et al. 2016), (Shen et al. 2015)); constrained optimal controllers (i.e. (Cuzzola et al.|2002), (Xia et al.|2008), (Lu et al.|2013)); and many others. The use of such dilation variables is conservative, thus it only provides sufficient conditions for the controller existence. To partially overcome this conservativeness, several authors have proposed the use of iterative methods to locally solve the resulting non-convex Bilinear Matrix Inequality (BMI) problem (i.e. (Sadeghzadeh and Momeni 2016), (Moreira et al. 2011), (Sadeghzadeh and Karimi 2015), (Peaucelle and Arzelier 2001), (Donkers 2017), (Sadeghzadeh 2014)). However, iterative methods also only provide sufficient conditions, due to its local optimality guarantees.

Another conservative aspect of robust synthesis and analysis methods is the assumption of an arbitrary (unstructured) norm bounded set since uncertainties are often known to be structured. Several studies investigated analysis and synthesis methods for linear systems under structured uncertainties. Shamma (Shamma 1994), Packard and Doyle (Packard and Doyle 1993), and Graham et al. (Graham et al. 2006) addressed the problem of robust stability analysis. Sznaier et al. (Sznaier et al. $\mid 2002)$, and Feron (Feron 1997) investigated robust $\mathrm{H}_{2}$ analysis. While $\mathrm{Wu}$ and $\mathrm{Lu}(\mathrm{Wu}$ and $\mathrm{Lu} 2004)$ addressed $H_{\infty}$ synthesis. However, according to Paganini (Paganini 2013$)$, the synthesis of a controller for systems subject to structured uncertainties results in non-convex optimizations.

In this paper, we propose a generalized Guaranteed Cost Control synthesis problem for discrete-time linear systems subject to norm-bounded structured parametric uncertainties for state-feedback and static output-feedback controllers. We consider a correlated block diagonal uncertainty structure, which is more general than previous studies (see for instance Szanaier et al. (Sznaier et al. 2002) and Graham et al. (Graham et al. 2006)). We also address the non-convexity issue discussed by Paganini through the use of the generalized S-Procedure, as proposed by Iwasaki et al. (Iwasaki et al. 2000), which is proved to be both necessary and sufficient for the considered structure. Meanwhile, we address the non-convexity of the output feedback synthesis problem conservatively through the dilated variable approach, based on the Reciprocal Projection Lemma (Apkarian et al. 2001).

This paper has the following organization: Section 2 presents the problem statement; Section 3 discusses preliminary results; Section 4 derives the proposed Guaranteed Cost Controller; Section 5 provides a numerical example; finally, Section 6 addresses the final remarks.

The notation used throughout this paper is standard. $I_{p}$ is a identity matrix of size

$p \times p, x^{T} M x=x^{T} M(\bullet)$ for brevity, $A \otimes B$ is the Kronecker product of matrices $A$ and $B$, and $\operatorname{tr}(A)$ is the trace of matrix $A$.

\section{Problem statement}

In this section, we present a Guaranteed Cost Controller definition with its required assumptions. We consider discrete-time linear system subject to parametric uncertain- 
ties of the form

$$
\begin{aligned}
x_{k+1} & =\left(A+\delta A\left(\Delta_{k}\right)\right) x_{k}+\left(B^{u}+\delta B^{u}\left(\Delta_{k}\right)\right) u_{k} \\
y_{k} & =\left(C_{y}+\delta C_{y}\left(\Delta_{k}\right)\right) x_{k},
\end{aligned}
$$

where $x_{k} \in \Re^{n_{x}}$ is the system state, $u_{k} \in \Re^{n_{u}}$ is the control input, $A \in \Re^{n_{x} \times n_{x}}$ is the state matrix, $B^{u} \in \Re^{n_{x} \times n_{u}}$ is the input matrix, $C_{y} \in \Re^{n_{y} \times n_{x}}$ is the output state matrix, and $\delta A\left(\Delta_{k}\right), \delta B^{u}\left(\Delta_{k}\right)$ and $\delta C_{y}\left(\Delta_{k}\right)$ are, respectively, the state, input and output multiplicative uncertainty matrices, such that

$$
\left[\begin{array}{cc}
\delta A\left(\Delta_{k}\right) & \delta B^{u}\left(\Delta_{k}\right) \\
\delta C_{y}\left(\Delta_{k}\right) & 0
\end{array}\right]=\left[\begin{array}{c}
B^{w} \\
D_{y}^{w}
\end{array}\right] \bar{\Delta}_{k}\left[\begin{array}{ll}
C_{z} & D_{z}^{u}
\end{array}\right]
$$

where $\bar{\Delta}_{k}=\Delta_{k}\left(I_{n_{q}}-D_{z}^{w} \Delta_{k}\right)^{-1}, \Delta_{k} \in \mathbb{D}^{u}=\left\{\Delta \mid \Delta \in \Re^{n_{p} \times n_{q}},\|\Delta\|_{2} \leq 1\right\}, B^{w} \in$ $\Re^{n_{x} \times n_{p}}, D_{y}^{w} \in \Re^{n_{y} \times n_{p}}, D_{z}^{w} \in \Re^{n_{q} \times n_{p}}, C_{z} \in \Re^{n_{q} \times n_{x}}$ and $D_{z}^{u} \in \Re^{n_{q} \times n_{u}}$.

In the scope of this paper, we are interested in investigating the controller synthesis when the structure of $\Delta_{k}$ is known. Therefore, we define $\mathbb{D} \subseteq \mathbb{D}^{u}$ to be the set of uncertainty matrices such that

$$
\begin{aligned}
\mathbb{D}=\left\{\Delta \mid \forall i \in[1, s]: \Delta_{i} \in \Re^{n_{p i} \times n_{q i}},\left\|\Delta_{i}\right\|_{2} \leq 1,\right. \\
\left.\Delta=\operatorname{diag}\left(I_{n_{r 1}} \otimes \Delta_{1}, I_{n_{r 2}} \otimes \Delta_{2}, \ldots, I_{n_{r s}} \otimes \Delta_{s}\right)\right\},
\end{aligned}
$$

where $n_{p}=\sum_{i=1}^{s} n_{r i} n_{p i}$ and $n_{q}=\sum_{i=1}^{s} n_{r i} n_{q i}$.

The uncertainty structure defined in (3) is more general than ones considered in previous studies. For example, the structure investigated by Sznaier et al. Sznaier et al. (2002) and Graham et al. Graham et al. (2006) is square block diagonal $\left(n_{r i}=1\right.$ and $\left.n_{p i}=n_{q i}\right)$.

Associated with the uncertain linear system (1) is the performance measurement cost function

$$
J\left(x_{0}\right)=\lim _{n \rightarrow \infty} \sum_{k=0}^{n} x_{k}^{T} Q x_{k}+2 x_{k}^{T} N u_{k}+u_{k}^{T} R u_{k},
$$

where $Q \in \Re^{n_{x} \times n_{x}}, N \in \Re^{n_{x} \times n_{u}}, R \in \Re^{n_{u} \times n_{u}}, Q \succeq 0, R \succ 0$, and

$$
\left[\begin{array}{cc}
Q & N \\
N^{T} & R
\end{array}\right]=\left[\begin{array}{c}
C_{c}^{T} \\
D_{c}^{u T}
\end{array}\right]\left[\begin{array}{ll}
C_{c} & D_{c}^{u}
\end{array}\right] \succeq 0
$$

and $C_{c} \in \Re^{n_{c} \times n_{x}}$ and $D_{c}^{u} \in \Re^{n_{c} \times n_{u}}$ denote the factorization of the cost function.

We are now ready to define the proposed Guaranteed Cost Controller.

Definition 2.1. A static output feedback controller $u_{k}=-K y_{k}$ is said to be a stabilizing Guaranteed Cost Controller for the uncertain system (1) if there exists a symmetric matrix $P \succ 0$ that upper-bounds the cost functional (4) for 


$$
\begin{aligned}
& {\left[A+\delta A+\left(B^{u}+\delta B^{u}\left(\Delta_{k}\right) K\left(C_{y}+\delta C_{y}\left(\Delta_{k}\right)\right]^{T} P[\bullet]-P+\right.\right.} \\
& Q+N K\left(C_{y}+\delta C_{y}\left(\Delta_{k}\right)\right)+\left(C_{y}+\delta C_{y}\left(\Delta_{k}\right)\right)^{T} K^{T} N^{T}+ \\
& +\left[K\left(C_{y}+\delta C_{y}\left(\Delta_{k}\right)\right)\right]^{T} R[\bullet] \leq 0
\end{aligned}
$$

such that $J\left(x_{0}\right) \leq J^{*}\left(x_{0}\right)=x_{0}^{T} P x_{0}$ for the closed loop system

$$
x_{k+1}=\left[A+\delta A\left(\Delta_{k}\right)-\left(B^{u}+\delta B^{u}\left(\Delta_{k}\right)\right) K\left(C_{y}+\delta C_{y}\left(\Delta_{k}\right)\right)\right] x_{k},
$$

and all admissible uncertainties $\Delta_{k} \in \mathbb{D}$.

Definition 2.2 (Optimal Guaranteed Cost Control). A static output-feedback controller $u_{k}=-K y_{k}$ is said to be optimal if it satisfies Definition 2.1 and $J^{*}\left(x_{0}\right)$ is minimal.

Remark 1. The static output-feedback GCC from Definition 2.1 reduces to the statefeedback case if $C_{y}=I_{n_{x}}, D_{y}^{w}=0$, and $\delta C_{y}\left(\Delta_{k}\right)=0$ for all $\Delta_{k} \in \mathbb{D}$.

The GCC problem from Definitions 2.1 and 2.2 is well posed under the following assumptions:

Assumption 2.3. For all $\Delta_{k} \in \mathbb{D}$, the pair $\left(A+\delta A\left(\Delta_{k}\right), B+\delta B\left(\Delta_{k}\right)\right)$ is stabilizable.

Assumption 2.4. The uncertainty matrices (2) are well-posed. Therefore, for all $\Delta_{k} \in \mathbb{D}, D_{z}^{w} \Delta_{k} \prec \mathbf{I}_{\mathbf{n}_{\mathbf{q}}}$, or equivalently, $\left\|D_{z}^{w}\right\|_{2}<1$.

Assumption 2.5. For all $\Delta_{k} \in \mathbb{D}$, the pair $\left(A+\delta A\left(\Delta_{k}\right), Q^{\frac{1}{2}}\right)$ is observable.

Assumption 2.6. For all $\Delta_{k} \in \mathbb{D}, D_{y}^{w} \bar{\Delta}_{k} D_{z}^{u}=0$. Therefore, the feed-through uncertainty is zero.

\section{Preliminary results}

This section presents previous results and preliminary work to formulate a convex condition for the Guaranteed Cost Controller. Such results include the generalized S-Procedure, the Reciprocal Projection Lemma, a property of the Kronecker Product, and an alternative representation of the system presented in Section 2 .

The uncertain linear system (1) is equivalent to

$$
\begin{aligned}
x_{k+1} & =A x_{k}+B^{w} w_{k}+B^{u} u_{k} \\
y_{k} & =C_{y} x_{k}+D_{y}^{w} w_{k} \\
z_{k} & =C_{z} x_{k}+D_{z}^{w} w_{k}+D_{z}^{u} u_{k},
\end{aligned}
$$

subject to the feedback disturbance $w_{k}=\Delta_{k} z_{k}$, where $w_{k} \in \Re^{n_{p}}$ and $z_{k} \in \Re^{n_{q}}$. Similarly, the closed loop system with a static output-feedback controller $u_{k}=-K y_{k}$ 
is equivalent to

$$
\begin{aligned}
x_{k+1} & =\left(A-B^{u} K C_{y}\right) x_{k}+\left(B^{w}-B^{u} K D_{y}^{w}\right) w_{k} \\
z_{k} & =\left(C_{z}-D_{z}^{u} K C_{y}\right) x_{k}+\left(D_{z}^{w}-D_{z}^{u} K D_{y}^{w}\right) w_{k}
\end{aligned}
$$

whose matrices will be denoted as $\bar{A}=A-B^{u} K C_{y}, \bar{B}^{w}=B^{w}-B^{u} K D_{y}^{w}, \bar{C}_{z}=$ $C_{z}-D_{z}^{u} K C_{y}$, and $\bar{D}_{z}^{w}=D_{z}^{w}-D_{z}^{u} K D_{y}^{w}$ for brevity. From (9), the closed loop uncertain system (7) is equivalent to

$$
x_{k+1}=\left(\bar{A}-\bar{B}^{w} \bar{\Delta}_{k} \bar{C}_{z}\right) x_{k},
$$

and the closed-loop cost function (4) can be rewritten as

$$
J\left(x_{0}\right)=\sum_{k=0}^{\infty} x_{k}^{T}\left(\bar{C}_{c}+\bar{D}_{c}^{w} \bar{\Delta}_{k} \bar{C}_{z}\right)^{T}(\bullet) x_{k},
$$

where $\bar{C}_{c}=C_{c}-D_{c} K C_{y}$, and $\bar{D}_{c}^{w}=-D_{c} K D_{y}^{w}$. Therefore, based on (10) and (11), the optimal GCC synthesis problem from Definition 2.2 is equivalent to

$$
\begin{aligned}
& J^{*}\left(x_{0}\right) \geq \max _{\Delta_{0}, \ldots, \Delta_{\infty}} \min _{K} J\left(x_{0}\right) \\
& \text { s.t. } x_{k+1}=\left(\bar{A}-\bar{B}^{w} \bar{\Delta}_{k} \bar{C}_{z}\right) x_{k} .
\end{aligned}
$$

\subsection{Generalized S-Procedure}

Iwasaki et al. (Iwasaki et al. 2000) described a generalized S-Procedure which provides both necessary and sufficient conditions for particular sets of matrices, entitled loss-

less sets. We now present the lossless set definition and the generalized S-Procedure theorem for completeness.

Definition 3.1 (Lossless Set). A subset $\mathbb{S}$ of $n \times n$ symmetric real matrices is said to be lossless if it has the following properties:

(i) $\mathbb{S}$ is convex;

(ii) $S \in \mathbb{S} \Rightarrow \forall \tau>0: \tau S \in \mathbb{S}$;

(iii) For each nonzero matrix $H \in \Re^{n \times n}, H \succeq 0$ such that $\forall S \in \mathbb{S}: \operatorname{tr}(S H) \leq 0$, there exist vectors $\lambda_{i} \in \Re^{n}$ such that

$$
H=\sum_{i=0}^{\operatorname{rank}(H)} \lambda_{i} \lambda_{i}^{*}, \forall S \in \mathbb{S}: \lambda_{i}^{*} S \lambda_{i} \leq 0 .
$$

Theorem 3.2 (Generalized S-Procedure). Let $\Theta \in \Re^{n \times n}$ be a symmetric matrix and $\mathbb{S}$ be a lossless set. Then, the following statements are equivalent:

(i) $\forall x \in \mathbb{X}=\left\{x \mid x \in \Re^{n}, x \neq 0, x^{T} S x \leq 0, \forall S \in \mathbb{S}\right\}: x^{T} \Theta x \leq 0$;

(ii) $\exists S \in \mathbb{S}: \Theta-S \preceq 0$.

Proof. See Theorem 1 of (Iwasaki et al. 2000). 


\subsection{Reciprocal projection lemma}

Apkarian et al. (Apkarian et al. 2001), based on Oliveira et. al. (de Oliveira et al. 1999b), proposed the Reciprocal Projection Lemma. Such Lemma enables several previously non-convex conditions present in synthesis problems to be posed as convex conditions through the use of additional slack variables. Based on this result less conservative methods have been developed, particularly in the field of multi-objective synthesis and analysis (i.e., (Köroğlu and Falcone 2014), (Adegas 2013)). We now present the Projection and the Reciprocal Projection Lemmas.

Lemma 3.3 (Projection Lemma). Let $\Psi \in \Re^{n \times n}$ be a symmetric matrix and $P, Q \in$ $\Re^{m \times n}$. Then, following statements are equivalent:

(i) There exists $X \in \Re^{m \times m}$ such that $\Psi+P^{T} X^{T} Q+Q^{T} X P \preceq 0$;

(ii) $N_{P}^{T} \Psi N_{P} \preceq 0$ and $N_{Q}^{T} \Psi N_{Q} \preceq 0$,

where $N_{P}$ and $N_{Q}$ are arbitrary bases of the null-space of $P$ and $Q$, respectively.

Proof. See Lemma 3.1 of (Gahinet and Apkarian 1994).

Lemma 3.4 (Reciprocal Projection Lemma). Let $\Psi \in \Re^{n \times n}$ be a symmetric matrix, $S \in \Re^{n \times n}$ and $X \in \Re^{n \times n}$ be an arbitrary positive definite matrix. Then, the following statements are equivalent:

(i) $\Psi+S+S^{T} \preceq 0$;

(ii) There exists $W \in \Re^{n \times n}$ such that

$$
\left[\begin{array}{cc}
-X & S+W \\
S^{T}+W^{T} & \Psi+X-W-W^{T}
\end{array}\right] \preceq 0
$$

Proof. See Lemma 2.2 of (Apkarian et al. 2001).

\subsection{Property of Kronecker products}

We also present a fundamental property of Kronecker product, necessary for the proposed controller proof.

Lemma 3.5. Let $X \in \Re^{p \times p}$ and $Y \in \Re^{q \times r}$. Then,

$$
Z=\left(X \otimes I_{q}\right)\left(I_{p} \otimes Y\right)=\left(I_{p} \otimes Y\right)\left(X \otimes I_{r}\right)
$$

Proof. This property follows directly from the fact that each block of $Z$ is given by $Z_{i, j}=X_{i, j} Y$, which is commutable since $X_{i, j}$ is a scalar.

Corollary 3.6. For all $i \in[1, n]$, let $X_{i} \in \Re^{p_{i} \times p_{i}}, Y_{i} \in \Re q_{i} \times r_{i}$, and

$$
Y=\operatorname{diag}\left(I_{p_{1}} \otimes Y_{1}, I_{p_{2}} \otimes Y_{2}, \ldots, I_{p_{n}} \otimes Y_{n}\right)
$$


Then,

$$
\operatorname{diag}\left(X_{1} \otimes I_{q_{1}}, X_{2} \otimes I_{q_{2}}, \ldots, X_{n} \otimes I_{q_{n}}\right) Y=Y \operatorname{diag}\left(X_{1} \otimes I_{r_{1}}, X_{2} \otimes I_{r_{2}}, \ldots, X_{n} \otimes I_{r_{n}}\right)
$$

follows directly from Lemma 3.5.

\section{Guaranteed cost control for systems with structured uncertainties}

In this section, we present the proposed static output-feedback controller. Existence condition for the particular case of no measured disturbance $D_{y}^{w}=0$ is first presented and we specialize it to the state-feedback controller. Then, we present the Optimal Static Output-Feedback Guaranteed Cost Controller developed.

Lemma 4.1 (Unstructured Uncertainty Set). Let $\xi_{k}=\left[x_{k}^{T}, w_{k}^{T}\right]^{T}$. Then, the region where $w_{k}=\Delta_{k} z_{k}$ holds for some $\Delta_{k} \in \mathbb{D}^{u}$ is

$$
\xi_{k} \in \mathbb{X}^{u}=\left\{\xi \mid \xi \in \Re^{n_{x}+n_{p}}, \xi^{T} S \xi \leq 0, \forall S \in \mathbb{S}^{u}\right\},
$$

where $\mathbb{S}^{u}=\left\{\lambda S^{u} \mid \lambda \geq 0\right\}$ is the unstructured uncertainty set, and

$$
S^{u}=\left[\begin{array}{cc}
\bar{C}_{z}^{T} \bar{C}_{z} & \bar{C}_{z}^{T} \bar{D}_{z}^{w} \\
\bar{D}_{z}^{w T} \bar{C}_{z} & \bar{D}_{z}^{w T} \bar{D}_{z}^{w}-I
\end{array}\right] .
$$

Proof. Direct from the discrete-time Bounded Real Lemma (de Souza and Xie 1992).

The set $\mathbb{S}^{u}$, from Lemma 4.1, has been thoroughly used for the analysis and synthesis of robust controllers. It defines the region of the domain of $\xi_{k}$ where we wish to ensure system stability and performance. In the case considered in this paper, the structure of the uncertainty $\Delta_{k}$ is known and can be used to provide a less conservative set, which still preserves the losslessness property from $\mathbb{S}^{u}$.

Lemma 4.2 (Structured Uncertainty Set). Let $\Lambda_{p}$, and $\Lambda_{q}$ be

$$
\begin{aligned}
& \Lambda_{p}=\operatorname{diag}\left(\Lambda_{1} \otimes I_{n_{p 1}}, \Lambda_{2} \otimes I_{n_{p 2}}, \ldots, \Lambda_{s} \otimes I_{n_{p s}}\right) \\
& \Lambda_{q}=\operatorname{diag}\left(\Lambda_{1} \otimes I_{n_{q 1}}, \Lambda_{2} \otimes I_{n_{q 2}}, \ldots, \Lambda_{s} \otimes I_{n_{q s}}\right)
\end{aligned}
$$

where for all $1 \leq i \leq s, \Lambda_{i} \in \Re^{n_{r i} \times n_{r i}}$ are positive semi-definite matrices. Then, the region where $w_{k}=\Delta_{k} z_{k}$ holds for some $\Delta_{k} \in \mathbb{D}$ is

$$
\xi_{k} \in \mathbb{X}=\left\{\xi \mid \xi \in \Re^{n_{x}+n_{p}}, \xi^{T} S \xi \leq 0, \forall S \in \mathbb{S}\right\},
$$

where $\mathbb{S}=\left\{S^{s}\left(\Lambda_{p}, \Lambda_{q}\right) \mid \forall i \in[1, s], \forall \Lambda_{i} \succeq 0\right\}$ is the structured uncertainty set, and

$$
S^{s}\left(\Lambda_{p}, \Lambda_{q}\right)=\left[\begin{array}{cc}
\bar{C}_{z}^{T} \Lambda_{q} \bar{C}_{z} & \bar{C}_{z}^{T} \Lambda_{q} \bar{D}_{z}^{w} \\
\bar{D}_{z}^{w T} \Lambda_{q} \bar{C}_{z} & \bar{D}_{z}^{w T} \Lambda_{q} \bar{D}_{z}^{w}-\Lambda_{p}
\end{array}\right]
$$


Proof. By left-multiplying the feedback disturbance equality $w_{k}=\Delta_{k} z_{k}$ by $\Lambda_{p}^{\frac{1}{2}}$, we obtain $\Lambda_{p}^{\frac{1}{2}} w_{k}=\Lambda_{p}^{\frac{1}{2}} \Delta_{k} z_{k}$. Which, based on Corollary 3.6 , is equivalent to

$$
\Lambda_{p}^{\frac{1}{2}} w_{k}=\Delta_{k} \Lambda_{q}^{\frac{1}{2}} z_{k}
$$

Consider the 2-norm of 23 . Then, for all $\Delta_{k} \in \mathbb{D}$,

$$
\left\|\Lambda_{p}^{\frac{1}{2}} w_{k}\right\|_{2}=\left\|\Delta_{k} \Lambda_{q}^{\frac{1}{2}} z_{k}\right\|_{2} \leq\left\|\Delta_{k}\right\|_{2}\left\|\Lambda_{q}^{\frac{1}{2}} z_{k}\right\|_{2} \leq\left\|\Lambda_{q}^{\frac{1}{2}} z_{k}\right\|_{2}
$$

which is equivalent to $w_{k}^{T} \Lambda_{p} w_{k}-z_{k}^{T} \Lambda_{q} z_{k} \leq 0$, or $\xi_{k}^{T} S^{s}\left(\Lambda_{p}, \Lambda_{q}\right) \xi_{k} \leq 0$. Therefore, (24) holds for all $\Lambda_{p} \succ 0, \Lambda_{q} \succ 0$, and $\Delta_{k} \in \mathbb{D}$, and the domain of $\xi_{k}$ with admissible feedback disturbance is defined by

$$
\xi_{k} \in \mathbb{X}=\left\{\xi \mid \xi \in \Re^{n_{x}+n_{p}}, \xi^{T} S \xi \leq 0, \forall S \in \mathbb{S}\right\} .
$$

Lemma 4.3. The Structured Uncertainty Set has the following properties:

(i) $\mathbb{S}^{u} \subseteq \mathbb{S}$;

(ii) The set $\mathbb{S}$ is lossless.

Proof. See Appendix A.

We are now ready to investigate the necessary and sufficient conditions for the existence of a GCC that satisfies Definition 2.1.

Theorem 4.4. Consider a static output-feedback controller $u_{k}=-K y_{k}$ regulating the System (1) subject to structured uncertainties of the form (3). Then, the following statements are equivalent:

(i) The controller satisfies Definition 2.1;

(ii) There exists a value function $V\left(x_{k}\right)=x_{k} P x_{k}$, where $P \succ 0$, such that for all $\Delta_{k} \in \mathbb{D}$

$$
V\left(x_{k+1}\right)-V\left(x_{k}\right) \leq-x_{k}^{T}\left(\bar{C}_{c}+\bar{D}_{c}^{w} \bar{\Delta}_{k} \bar{C}_{z}\right)^{T}(\bullet) x_{k}
$$

(iii) There exists $P \succ 0$ and $\Lambda_{i} \succeq 0$ for $i \in[1, s]$ such that

$$
\begin{aligned}
{\left[\begin{array}{cc}
\bar{A}^{T} P \bar{A}-P & \bar{A}^{T} P \bar{B}^{w} \\
\bar{B}^{w T} P \bar{A} & \bar{B}^{w T} P \bar{B}^{w}
\end{array}\right]+} & {\left[\begin{array}{cc}
\bar{C}_{c}^{T} \bar{C}_{c} & \bar{C}_{c}^{T} \bar{D}_{c}^{w} \\
\bar{D}_{c}^{w T} \bar{C}_{c} & \bar{D}_{c}^{w T} \bar{D}_{c}^{w T}
\end{array}\right]+} \\
& +\left[\begin{array}{cc}
\bar{C}_{z}^{T} \Lambda_{q} \bar{C}_{z} & \bar{C}_{z}^{T} \Lambda_{q} \bar{D}_{z}^{w} \\
\bar{D}_{z}^{w T} \Lambda_{q} \bar{C}_{z} & \bar{D}_{z}^{w T} \Lambda_{q} \bar{D}_{z}^{w T}-\Lambda_{p}
\end{array}\right] \preceq 0 .
\end{aligned}
$$

Proof. We first prove the equivalnece of (i) and (ii), then we prove the equivalence between (ii) and (iii). 
(i) $\Rightarrow$ (ii): Let $c\left(x_{k}, u_{k}\right)=x_{k}^{T} Q x_{k}+2 x_{k}^{T} N u_{k}+u_{k}^{T} R u_{k}$ for brevity and consider the value function $V_{k}^{*}\left(x_{k}\right)$. From Bellman's optimality principle, we obtain

$$
V_{k}^{*}\left(x_{k}\right)=\max _{\Delta_{k} \in \mathbb{D}} \min _{u_{k}} c\left(x_{k}, u_{k}\right)+V_{k+1}^{*}\left(x_{k+1}\right) .
$$

In the infinite horizon case, 28 becomes

$$
V^{*}\left(x_{k}\right)=\max _{\Delta_{k} \in \mathbb{D}} \min _{u_{k}} c\left(x_{k}, u_{k}\right)+V^{*}\left(x_{k+1}\right) .
$$

Assume (i) holds, then from Definition 2.1 there exists a stabilizing controller $u_{k}=$ $-K y_{k}$ and a sub-optimal value function $V\left(x_{k}\right)=x_{k}^{T} P x_{k} \geq V^{*}\left(x_{k}\right)$ such that

$$
V\left(x_{k}\right)=\max _{\Delta_{k} \in \mathbb{D}} c\left(x_{k},-K y_{k}\right)+V\left(x_{k+1}\right)
$$

which implies

$$
\forall \Delta_{k} \in \mathbb{D}: V\left(x_{k}\right) \geq c\left(x_{k},-K y_{k}\right)+V\left(x_{k+1}\right) .
$$

Therefore, (31) holds for the quadratic form and is equivalent to (ii), since $c\left(x_{k},-K y_{k}\right)=x_{k}^{T}\left(\bar{C}_{c}+\bar{D}_{c}^{w} \bar{\Delta}_{k} C_{z}\right)^{T}(\bullet) x_{k}$.

(i) $\Leftarrow$ (ii): Now assume (i) does not holds, then there isn't a sub-optimal value function with quadratic form that upper-bounds $(29)$. In such case, the infinite horizon Bellman's optimality principle does not have a solution. Therefore, (ii) also doesn't hold.

(ii) $\Rightarrow$ (iii): Assume (ii) holds and consider the system model (9), then (31) is equivalent to $\xi_{k}^{T} M \xi_{k} \leq 0$, where

$$
M=\left[\begin{array}{cc}
\bar{A}^{T} P \bar{A}-P & \bar{A}^{T} P \bar{B}^{w} \\
\bar{B}^{w T} P \bar{A} & \bar{B}^{w T} P \bar{B}^{w}
\end{array}\right]+\left[\begin{array}{cc}
\bar{C}_{c}^{T} \bar{C}_{c} & \bar{C}_{c}^{T} \bar{D}_{c}^{w} \\
\bar{D}_{c}^{w T} \bar{C}_{c} & \bar{D}_{c}^{w T} \bar{D}_{c}^{w T}
\end{array}\right]
$$

for all $\Delta_{k} \in \mathbb{D}$. From Lemma 4.2, (32) holds for all $\xi \in \mathbb{X}$. Then, based on the generalized S-Procedure, there exists $S \in \mathbb{S}$ such that $M-S \preceq 0$. Or equivalently, there exists $\Lambda_{i} \succeq 0$ for $i \in[1, s]$ such that (27) holds. Therefore, (iii) holds.

(ii) $\Leftarrow$ (iii): Conversely, if (ii) does not hold, there doesn't exist a $P \succ 0$ such that (32) holds. Therefore (iii) also does not hold.

\subsection{Convex condition for systems without disturbance feed-through}

If $D_{y}^{w}=0$ is assumed, we obtain $\bar{B}^{w}=B^{w}, \bar{D}_{z}^{w}=D_{z}^{w}$, and $\bar{D}_{c}^{w}=\mathbf{0}_{\mathbf{n}_{\mathbf{c}} \times \mathbf{n}_{\mathbf{p}}}$. In such a case, a simplified existence condition can be obtained.

Lemma 4.5. Assume $D_{y}^{w}=0$. Then, a static output-feedback controller $u_{k}=-K y_{k}$ is said to be of guaranteed cost, according to Definition 2.1, if and only if there exists 
$X \succ 0$ and $\Upsilon_{i} \succeq 0$ for $i \in[1, s]$ such that

$$
\left[\begin{array}{ccccc}
-\Upsilon_{q} & 0 & 0 & C_{z} X-D_{z}^{u} Y C_{y} & D_{z}^{w} \Upsilon_{p} \\
\star & -I_{n_{c}} & 0 & C_{c} X-D_{c}^{u} Y C_{y} & 0 \\
\star & \star & -X & A X-B^{u} Y C_{z} & B^{w} \Upsilon_{p} \\
\star & \star & \star & -X & 0 \\
\star & \star & \star & \star & -\Upsilon_{p}
\end{array}\right] \preceq 0
$$

where $\bar{X} C_{y}=C_{y} X$ and $Y=K \bar{X}$.

Proof. We first apply the Schur complement in $(27)$ to the terms dependent on $P$, to the cost terms, and to the terms dependent on $\Lambda_{q}$, which results

$$
\left[\begin{array}{ccccc}
-\Lambda_{q}^{-1} & 0 & 0 & \bar{C}_{z} & D_{z}^{w} \\
\star & -I_{n_{c}} & 0 & \bar{C}_{c} & 0 \\
\star & \star & -P^{-1} & \bar{A} & B^{w} \\
\star & \star & \star & -P & 0 \\
\star & \star & \star & \star & -\Lambda_{p}
\end{array}\right] \preceq 0
$$

Then, we apply the congruence transformation in (34) with $T=$ $\operatorname{diag}\left(I_{n_{q}}, I_{n_{c}}, I_{n_{x}}, P^{-1}, \Lambda_{p}^{-1}\right)$, and perform the substitution $X=\stackrel{P^{-1}}{\Upsilon_{q}} \Upsilon_{p}=\Lambda_{p}^{-1}$, and $\Upsilon_{q}=\Lambda_{q}^{-1}$, obtaining

$$
\left[\begin{array}{ccccc}
-\Upsilon_{q} & 0 & 0 & \bar{C}_{z} X & D_{z}^{w} \Upsilon_{p} \\
\star & -I_{n_{c}} & 0 & \bar{C}_{c} X & 0 \\
\star & \star & -X & \bar{A} X & B^{w} \Upsilon_{p} \\
\star & \star & \star & -X & 0 \\
\star & \star & \star & \star & -\Upsilon_{p}
\end{array}\right] \preceq 0
$$

Based on the substitutions $\bar{X} C_{y}=C_{y} X, Y=K \bar{X}, \bar{A} X=A X-B^{u} Y C_{z}, \bar{C}_{c} X=$ $C_{c} X-D_{c}^{u} Y C_{y}$, and $\bar{C}_{z} X=C_{z} X-D_{z}^{u} Y C_{y}$, (33) and (35) are equivalent.

Remark 2. The control gain can be recovered from Lemma 4.5 by $K=Y\left(C_{y} X C_{y}^{\dagger}\right)^{-1}$, where $C_{y}^{\dagger}$ is the pseudo-inverse of $C_{y}$.

Remark 3. Given $C_{y}=I_{n_{x}}$, we obtain $X=\bar{X}, K=Y X^{-1}$, and Lemma 4.5 reduces to the state-feedback controller synthesis condition.

Lemma 4.5 provides an LMI condition for the existence of the GCC, based on the multipliers $\Lambda_{i}$ for $i \in[1, s]$, the inverse cost function matrix $X$, and $Y$. From the properties of LMIs we can conclude that $(33)$ is convex.

\subsection{Convex condition for systems with disturbance feed-through}

If we consider the general case, where $D_{y}^{w} \neq 0$, the controller gain $K$ would also be present in the fifth column of (33). In such a case, the substitution which yields $Y$, used in Lemma 4.5, would not make the condition linear since $K$ would still exist in the fifth row and column. 
The following Theorem is the main result of this paper. It demonstrates that a similar substitution can be successfully used to convexify a "dilated" version of condition (33), based on the Reciprocal Projection Lemma 3.4.

Theorem 4.6. A static output-feedback controller $u_{k}=-K y_{k}$ is said to be of guaranteed cost, according to Definition 2.1. if there exists $X \succ 0, \Lambda_{i} \succeq 0$ for $i \in[1, s]$, $V_{2,2} \in \Re^{n_{c} \times n_{c}}, V_{3,3} \in \Re^{n_{x} \times n_{x}}, V_{4,4} \in \Re^{n_{x} \times n_{x}}, V_{4,5} \in \Re^{n_{x} \times n_{q}}, V_{5,4} \in \Re^{n_{q} \times n_{x}}$, $V_{5,5} \in \Re^{n_{q} \times n_{q}}$, and $Y \in \Re^{n_{u} \times n_{y}}$ such that

$$
\left[\begin{array}{ccc}
-M & 0 & V \\
\star & -M & \bar{N}+M \\
\star & \star & -V-V^{T}
\end{array}\right] \preceq 0
$$

where, $M=\operatorname{diag}\left(\Upsilon_{q}, I_{n_{c}}, X, X, \Upsilon_{q}\right)$,

$$
\begin{aligned}
& V=\left[\begin{array}{ccccc}
V_{1,1} & V_{1,2} & V_{1,3} & V_{1,4} & V_{1,5} \\
V_{2,1} & V_{2,2} & V_{2,3} & V_{2,4} & V_{2,5} \\
V_{3,1} & V_{3,2} & V_{3,3} & V_{3,4} & V_{3,5} \\
0 & 0 & 0 & V_{4,4} & V_{4,5} \\
0 & 0 & 0 & V_{5,4} & V_{5,5}
\end{array}\right] \\
& \bar{N}=\frac{1}{2}\left[\begin{array}{ccccc}
-V_{1,1} & -V_{1,2} & -V_{1,3} & \Phi_{z} & \Sigma_{z} \\
-V_{2,1} & -V_{2,2} & -V_{2,3} & \Phi_{c} & \Sigma_{c} \\
-V_{3,1} & -V_{3,2} & -V_{3,3} & \Phi_{x} & \Sigma_{x} \\
0 & 0 & 0 & -V_{4,4} & -V_{4,5} \\
0 & 0 & 0 & -V_{5,4} & -V_{5,5}
\end{array}\right] \\
& {\left[\begin{array}{cc}
\Phi_{z} & \Sigma_{z} \\
\Phi_{c} & \Sigma_{c} \\
\Phi_{x} & \Sigma_{x}
\end{array}\right]=2\left[\begin{array}{cc}
C_{z} & D_{z}^{w} \\
C_{c} & 0 \\
A & B^{u}
\end{array}\right]\left[\begin{array}{ll}
V_{4,4} & V_{4,5} \\
V_{5,4} & V_{5,5}
\end{array}\right]+\left[\begin{array}{cc}
V_{1,4} & V_{1,5} \\
V_{2,4} & V_{2,5} \\
V_{3,4} & V_{3,5}
\end{array}\right]-2\left[\begin{array}{c}
D_{z}^{u} \\
D_{c}^{u} \\
B^{u}
\end{array}\right] Y\left[\begin{array}{ll}
C_{y} & D_{y}^{w}
\end{array}\right]} \\
& \bar{V}\left[\begin{array}{ll}
C_{y} & D_{y}^{w}
\end{array}\right]=\left[\begin{array}{ll}
C_{y} & D_{y}^{w}
\end{array}\right]\left[\begin{array}{ll}
V_{4,4} & V_{4,5} \\
V_{5,4} & V_{5,5}
\end{array}\right], \\
& Y=K \bar{V}, X=P^{-1}, \Upsilon_{p}=\Lambda_{p}^{-1} \text {, and } \Upsilon_{q}=\Lambda_{q}^{-1} \text {. }
\end{aligned}
$$

Proof. We apply again Schur complement in 27) to obtain

$$
\left[\begin{array}{ccccc}
-\Lambda_{q}^{-1} & 0 & 0 & \bar{C}_{z} & \bar{D}_{z}^{w} \\
\star & -I_{n_{c}} & 0 & \bar{C}_{c} & \bar{D}_{c}^{w} \\
\star & \star & -P^{-1} & \bar{A} & \bar{B}^{w} \\
\star & \star & \star & -P & 0 \\
\star & \star & \star & \star & -\Lambda_{p}
\end{array}\right] \preceq 0 .
$$

Then, we apply the congruence transformation in (41) with $T=$ 
$\operatorname{diag}\left(\lambda_{q}, I_{n_{c}}, P, I_{n_{x}}, I_{n_{p}}\right)$, and obtain

$$
E=\left[\begin{array}{ccccc}
-\Lambda_{q} & 0 & 0 & \Lambda_{q} \bar{C}_{z} & \Lambda_{q} \bar{D}_{z}^{w} \\
\star & -I_{n_{c}} & 0 & \bar{C}_{c} & \bar{D}_{c}^{w} \\
\star & \star & -P & P \bar{A} & P \bar{B}^{w} \\
\star & \star & \star & -P & 0 \\
\star & \star & \star & \star & -\Lambda_{p}
\end{array}\right] \preceq 0 .
$$

We now define $S$ as

$$
S=\left[\begin{array}{ccccc}
-\frac{1}{2} \Lambda_{q} & 0 & 0 & \Lambda_{q} \bar{C}_{z} & \Lambda_{q} \bar{D}_{z}^{w} \\
0 & -\frac{1}{2} I_{n_{c}} & 0 & \bar{C}_{c} & \bar{D}_{c}^{w} \\
0 & 0 & -\frac{1}{2} P & P \bar{A} & P \bar{B}^{w} \\
0 & 0 & 0 & -\frac{1}{2} P & 0 \\
0 & 0 & 0 & 0 & -\frac{1}{2} \Lambda_{p}
\end{array}\right]
$$

such that $S+S^{T}=E$. Then, from the Reciprocal Projection Lemma (Lemma 3.4) we obtain that for any given $Y \succ 0$ there exists $W$, of appropriate dimensions, such that

$$
\left[\begin{array}{cc}
-Y & S+W \\
\star & Y-W-W^{T}
\end{array}\right] \preceq 0 .
$$

Subsequently, we apply a congruence transformation in 44 with $T=$ $\operatorname{diag}\left(M, I_{n_{q}+n_{c}+n_{x}+n_{x}+n_{p}}\right)$ and substitute $Y=M^{-1}$, which yields

$$
\left[\begin{array}{cc}
-M & M S+M W \\
\star & M^{-1}-W-W^{T}
\end{array}\right] \preceq 0 .
$$

Let $V=W^{-1}$ and assume it takes the form (37). Then, we apply a congruence transformation in 44 with $T=\operatorname{diag}\left(I_{n_{q}+n_{c}+n_{x}+n_{x}+n_{p}}, V\right)$, resulting

$$
\left[\begin{array}{cc}
-M & M S V+M \\
\star & V M^{-1} V-V-V^{T}
\end{array}\right] \preceq 0
$$

which, with a Schur complement of the terms related to $M^{-1}$ and $M S V=\bar{N}$, is equivalent to

$$
\left[\begin{array}{ccc}
-M & 0 & V \\
\star & -M & \bar{N}+M \\
\star & \star & -V-V^{T}
\end{array}\right] \preceq 0
$$

which, in turn, is identical to (36).

Theorem 4.6 provides a convex condition for the existence of the GCC in the general static output-feedback case. It enables the synthesis of Optimal GCCs through SDP optimization problems. 


\subsection{Optimal static Output-feedback guaranteed cost control synthesis}

In this sub-section, we present the resulting optimization problem for the optimal static output feedback GCC synthesis. Although the formulated GCC conditions are stateindependent, the cost function (4) is still dependent. Therefore, we must represent the system cost in a state-independent manner. Two methods are often employed to address this issue: minimizing the worst case cost for all states in the unit norm ball (Petersen et al. 1998), or the expected cost value for a zero mean and unit covariance initial state distribution (Xie and Soh 1993). We have chosen the latter, since the worst case cost only minimizes the largest eigenvalue of $P$, while the stochastic interpretation minimizes $\operatorname{tr}(P)$. Another advantage of such an approach is its equivalence to the LQR when the system reduces to the state-feedback case without uncertainties.

Assumption 4.7. The initial state $x_{0}$ is a zero mean random variable with unit covariance.

From Assumption 4.7, $E\left(J^{*}\left(x_{0}\right)\right)=E\left(x_{0}^{T} P x_{0}\right)=E\left(\operatorname{tr}\left(x_{0}^{T} P x_{0}\right)\right)=\operatorname{tr}\left(P E\left(x_{0} x_{0}^{T}\right)\right)=$ $\operatorname{tr}(P)$.

Theorem 4.8. A static output feedback controller $u_{k}=-K y_{k}$ is said to be an optimal guaranteed cost, according to Definition 2.2, if and only if

$$
\begin{aligned}
K=\arg \min & \operatorname{tr}(Z) \\
\text { s.t. } & {\left[\begin{array}{ccc}
-M & 0 & V \\
\star & -M & \bar{N}+M \\
\star & \star & -V-V^{T}
\end{array}\right] \preceq 0 } \\
& {\left[\begin{array}{cc}
-Z & I \\
\star & -X
\end{array}\right] \preceq 0 }
\end{aligned}
$$

where $M, \bar{N}, V$ and $X$ are defined in Theorem 4.6, and $Z \in \Re^{n_{x} \times n_{x}}$ is the cost matrix $P$ relaxation.

Proof. From Definition 2.2, a controller is of optimal guaranteed cost if it satisfies Definition 2.1 and $J^{*}\left(x_{0}\right)$ is minimal. Since the distribution of $x_{0}$ is assumed to be known (Assumption 4.7), consider $\widehat{J}=E\left(J\left(x_{0}\right)\right)$. Then, the optimal GCC synthesis is equivalent to

$$
\begin{aligned}
\widehat{J}^{*}=\min & \operatorname{tr}(P) \\
\text { s.t. } & {\left[\begin{array}{ccc}
-M & 0 & V \\
\star & -M & \bar{N}+M \\
\star & \star & -V-V^{T}
\end{array}\right] \preceq 0 }
\end{aligned}
$$

which minimizes the expected cost. However, such optimization is not linear on $P$ since the LMI depends on its inverse $X=P^{-1}$. Therefore, we introduce the relaxation variable $Z \in \Re^{n_{x} \times n_{x}}$, such that $Z \succeq P$. From Schur complement, we obtain

$$
\left[\begin{array}{cc}
-Z & I \\
\star & -X
\end{array}\right] \preceq 0
$$

Notice that minimizing $\mathrm{Z}$ is equivalent to minimizing $P$, since its constraint is only dependent on $X$. Therefore, 49 is equivalent to 48. 
Remark 4. The optimal static output feedback for the case $D_{y}^{w}=0$, can be defined similarly to Theorem 4.6, with the substitution of condition (36) by (33).

\section{Numerical examples}

This section provides two numerical examples of GCC for systems subject to structured uncertainties. The first compares the proposed synthesis method with GCC for systems subject to unstructured uncertainties Xie and Soh (1993), and the Linear Quadratic Regulator Kalman et al. (1960). The second presents the synthesis and simulation for a system that previously proposed methods cannot synthesize a controller. We have used the YALMIP Toolbox Löfberg (2004) and the Mosek solver ApS (2015) for the modeling of the problem ${ }^{1}$.

\subsection{Example 1}

Consider the uncertain linear system ((1) and (2)), from Terra et al. (2014), defined by

$$
\begin{gathered}
A=\left[\begin{array}{ccc}
1.1 & 0 & 0 \\
0 & 0 & 1.2 \\
-1 & 1 & 0
\end{array}\right], B^{u}=\left[\begin{array}{cc}
0 & 1 \\
1 & 1 \\
-1 & 0
\end{array}\right], B^{w}=\left[\begin{array}{cc}
0.7 & 0.3 \\
0.5 & -0.4 \\
-1 & 0
\end{array}\right], \\
C_{z}=\left[\begin{array}{ccc}
0.41 & 0.43 & -0.5 \\
0 & -0.32 & 0.44
\end{array}\right], D_{z}^{u}=\left[\begin{array}{cc}
0.4 & -0.4 \\
0 & 0
\end{array}\right], D_{z}^{w}=0
\end{gathered}
$$

measurement matrices

$$
C_{y}=I_{n_{x}}, D_{y}^{w}=0
$$

and the disturbance

$$
\Delta_{k}=\left[\begin{array}{cc}
\delta_{1, k} & 0 \\
0 & \delta_{2, k}
\end{array}\right]
$$

where $\delta_{1, k}, \delta_{1, k} \in[-1,1]$. In this example, we synthesize and compare controllers for five different methods.

(1) LQR Kalman et al. (1960): The LQR controller is generated based on the nominal plant with no modeled disturbances.

(2) GCC (unstructured uncertainty) Xie and Soh (1993): Based on Xie's approach.

(3) Theorem 4.6 based GCC (unstructured): Controller based on Theorem 4.6, generated with the SDP optimization of Theorem 4.8, while assuming no structure to $\Delta_{k}$. Therefore, $\Lambda_{p}=\lambda I_{p}$ and $\Lambda_{q}=\lambda I_{q}$ where $\lambda>0$.

(4) Lemma 4.5-based GCC (structured): Controller based on Theorem 4.5, generated with the SDP optimization of Theorem 4.8 without the "Dilated" LMI (see Remark (4), while exploiting the diagonal uncertainty structure of $\Delta_{k}$. Therefore, $\Lambda_{p}=\operatorname{diag}\left(\lambda_{1}, \lambda_{2}\right)$ and $\Lambda_{q}=\operatorname{diag}\left(\lambda_{1}, \lambda_{2}\right)$ where $\lambda_{i}>0$ for all $i \in[1,2]$.

\footnotetext{
${ }^{1}$ The source code for both numerical examples are available at: https://gitlab.com/cmasseraf/gcc structured_uncertainty
} 


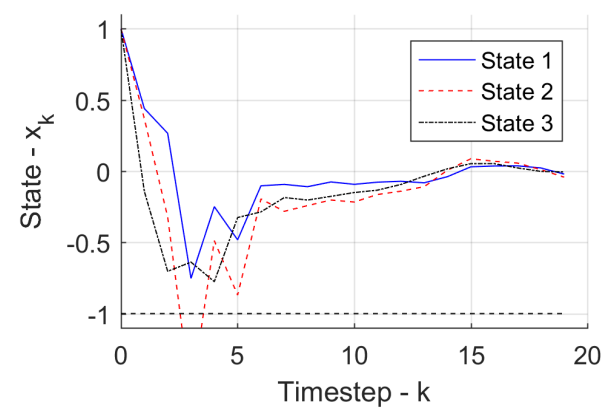

(a)

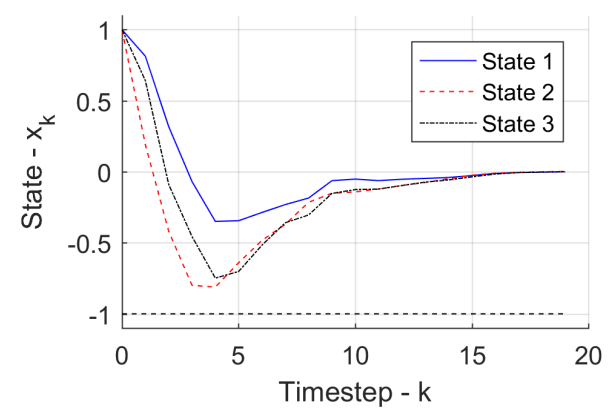

(c)

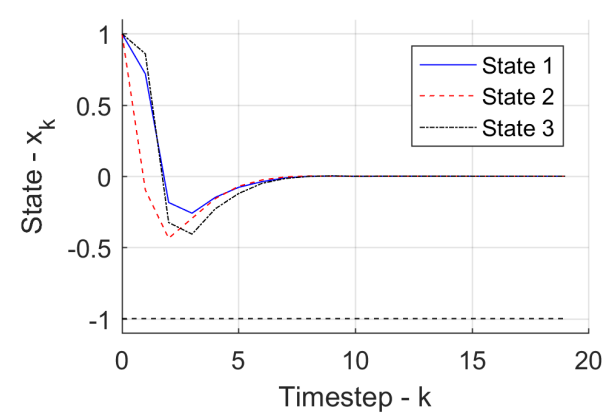

(e)

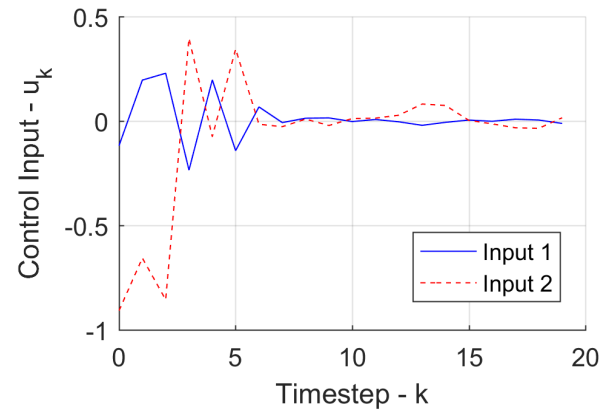

(b)

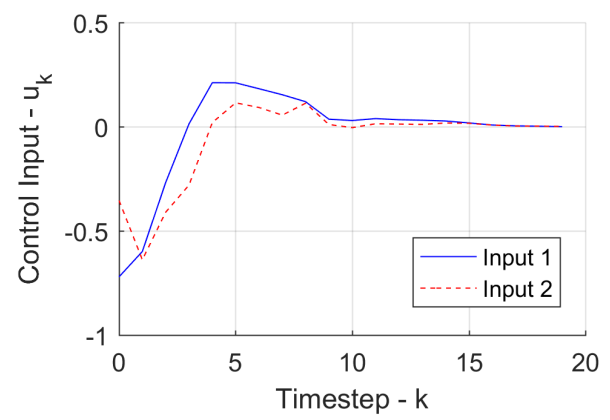

(d)

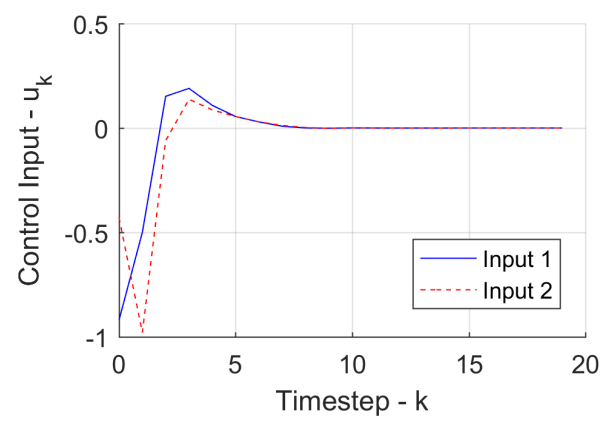

(f)

Figure 1.: Simulation results for the synthesized results of Example 1. (a) Closed-loop state $x_{k}$ subject to uncertainties for (1); (b) Control inputs $u_{k}$ for (1); (c) Closed-loop state $x_{k}$ subject to uncertainties for (2) and (3); (d); Control inputs $u_{k}$ for (2) and (3); (e) Closed-loop state $x_{k}$ subject to uncertainties for (4) and (5); (f) Control inputs $u_{k}$ for (4) and (5).

(5) Theorem 4.6-based GCC (structured): Controller based on Theorem 4.6, generated with the SDP optimization of Theorem 4.8, while exploiting the diagonal uncertainty structure.

The cost function matrices selected for all methods were $Q=I_{3}$, and $R=I_{2}$. Different methods are compared based on the synthesis cost, $\operatorname{tr}(P)$, and the effective cost, $E\left(J\left(x_{0}\right)\right)$. We calculated the effective cost as the mean value of the cost function for 5000 simulations performed with a horizon of 200 time-steps. Then, we present a simulation example for three controllers synthesized from these methods and qualitatively compare the obtained results. 
Table 1 presents the results of Example 1. It is possible to see that for this particular system, the effective cost of the LQR (1) exceeds its synthesis cost by one order of magnitude demonstrating its expected lack of robustness margins, although it is still able to stabilize the system. Both unstructured uncertainty GCCs (2 and 3) yield the same control gain and cost since $D_{y}^{w}=0$. Their synthesis cost is greater than the effective cost, which illustrates the upper-bound nature of its robustness margins. However, they yield extremely conservative bounds on cost (of an order of magnitude) due to not exploiting the uncertainty structure. Finally, both structured uncertainty GCCs (4 and 5) also yield the same control gain to each other since $D_{y}^{w}=0$, but decrease the conservativeness of the synthesis by a factor of seven while also reducing the effective cost from 46.56 to 44.16 .

Table 1.: Synthesis and simulation results for the comparative study

\begin{tabular}{|l|c|c|}
\cline { 2 - 3 } \multicolumn{1}{c|}{} & Synthesis cost & Effective cost \\
\hline 1) LQR & 22.15 & 490.13 \\
\hline 2) GCC proposed by Xie & 581.79 & 46.56 \\
\hline 3) Theorem 4.6 GCC (1996) & 581.79 & 46.56 \\
\hline 4) Lemma 4.5 GCC (structured) & 97.63 & 44.16 \\
\hline 5) Theorem 4.6 GCC (structured) & 97.63 & 44.16 \\
\hline
\end{tabular}

Figure 1 presents results acquired by one of the 5000 simulations performed with the set of controllers considered in this example. Identical disturbances and initial states, $x_{0}=[1,1,1]^{T}$, were used for all simulation. Figures $1 \mathrm{a}$ and $1 \mathrm{~b}$ show the results for the standard LQR controller with effective cost 12.6707. We can observe a significant impact of the disturbances on the overall system behavior, due to the lack of robustness guarantees on its synthesis. Figures $1 \mathrm{c}$ and $1 \mathrm{~d}$ show the results for the GCC considering unstructured disturbances with effective cost 10.7861; we can observe that it rejects the disturbances significantly better than the LQR controller; however, it yields an $80 \%$ overshoot before reaching steady-state. Finally, Figures $1 \mathrm{e}$ and $1 \mathrm{f}$ show the results for the GCC considering unstructured disturbances with effective cost of 7.3934. This controller was able to yield a faster settling time, smaller overshoot, and lower effective cost when compared to both other synthesized controllers.

The average cost reduction, combined by the performance improvement observed in Figure 1, demonstrates the advantages of incorporating the uncertainty structure information into the controller synthesis procedure.

\subsection{Example 2}

In this example we present the synthesis and simulation for a system that was not supported by previous methods, where $D_{y}^{w} \neq 0$. Consider the system from (51) with measurement matrices

$$
C_{y}=\left[\begin{array}{lll}
1 & 0 & 0 \\
0 & 1 & 0 \\
0 & 0 & 1 \\
0 & 0 & 0
\end{array}\right], D_{y}^{w}=\left[\begin{array}{ll}
0 & 0 \\
0 & 0 \\
0 & 0 \\
0 & 1
\end{array}\right] .
$$

In such a case, the LQR (1), Xie's GCC (2) and Lemma 4.5-based GCC (4) cannot be used as they assume $D_{y}^{w}=0$. However, the unstructured uncertainty GCC based 


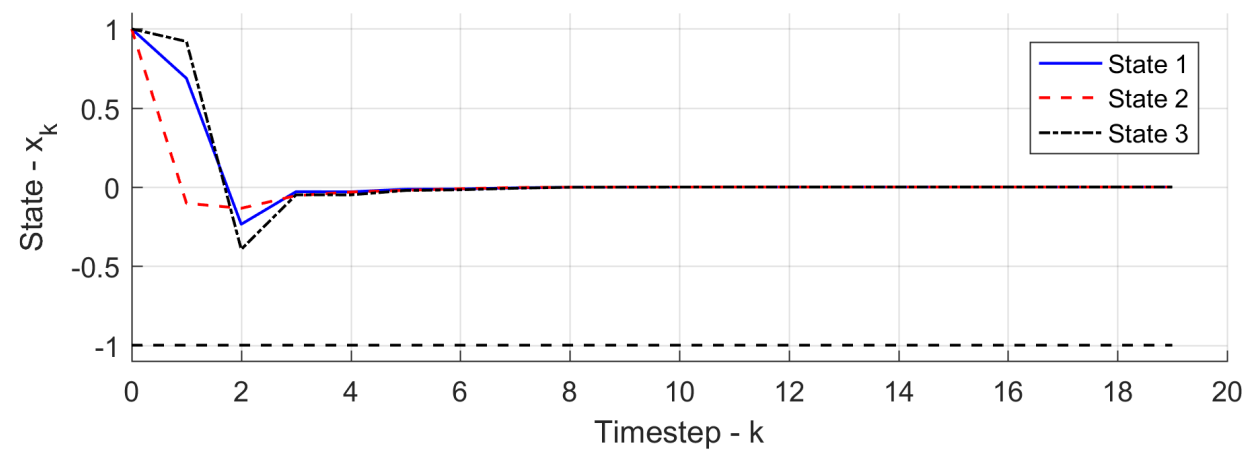

(a)

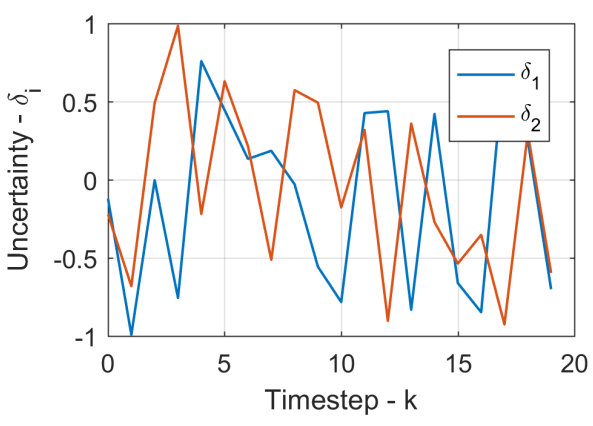

(b)

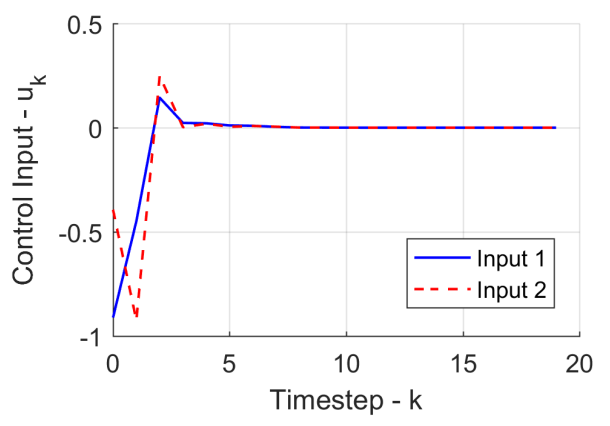

(c)

Figure 2.: Simulation results for GCC of Example 2. (a) Closed-loop state $x_{k}$ subject to uncertainties; (b) Simulated uncertainty $\delta_{1}$ and $\delta_{2}$; (c) Control inputs $u_{k}$.

on Theorem 4.6 (3) also cannot be synthesized, since Assumption 2.6 $\left(D_{y}^{w} \Delta_{k} D_{z}^{u}=0\right)$ does not hold for the generic uncertainty case. Therefore, the proposed GCC controller based on Theorem 4.6 for structured uncertainties is the only capable of synthesizing a controller for this system.

The structured uncertainty GCC based on Theorem 4.6 synthesis resulted in a gain matrix

$$
K=\left[\begin{array}{cccc}
1.1431 & 0.1282 & -0.3585 & 0.0947 \\
0.6881 & -0.7581 & 0.4561 & 0.0596
\end{array}\right]
$$

and cost matrix

$$
P=\left[\begin{array}{ccc}
61.9182 & 3.2483 & -41.2619 \\
3.2483 & 9.8246 & -7.1655 \\
-41.2619 & -7.1655 & 34.2462
\end{array}\right]
$$

It provides a synthesis cost of 94.15 and an effective cost of 45.62. Figure 2 shows the simulation result for initial state $x_{0}=[1,1,1]^{T}$, where we can observe that the control law is able to correctly stabilize the system subject to varying disturbances. 


\section{Conclusion}

In this paper, we have proposed a state-feedback and static output-feedback generalized Guaranteed Cost Control method for discrete-time linear systems subject to normbounded structured parametric uncertainties. Such controllers provide upper bounds to the quadratic cost functional and guarantee robust stability and performance to a larger class of uncertain linear systems if compared with previous methods. There are two main contributions for the class of systems considered: It relaxes the assumption from no uncertainty feed-through $\left(D_{y}^{w}=0\right)$ to an assumption that no feed-through uncertainty is multiplicative with control inputs ones $\left(D_{y}^{w} \Delta_{k} D_{z}^{u}=0\right)$. Moreover, it represents a more general uncertainty structure (correlated block diagonal).

Numerical simulations exemplified that exploiting the internal structure of uncertainties may provide a significant reduction in conservativeness while still yielding controllers which guarantee robust stability and performance.

Although the proposed method provides both necessary and sufficient conditions for the synthesis of GCCs with a more general uncertainty structure, it still provides only sufficient conditions for the synthesis of robust static output-feedback controllers. Besides the underlying limitation that Guaranteed Cost Controllers only provide sufficient conditions for the optimal controller robust synthesis due to the simplifying assumption $P(\Delta)=P$ on the structure of the cost function.

As future works, we intend to include the extension of such method for constrained linear uncertain systems, hybrid systems and its application to mobile robotic system control.

\section{References}

Adegas, F.D., 2013. New sufficient LMI conditions for static output stabilization, IFAC Proceedings Volumes, 46 (2), 184-189.

Apkarian, P., Tuan, H.D., and Bernussou, J., 2001. Continuous-time analysis, eigenstructure assignment, and $H_{2}$ synthesis with enhanced LMI characterizations, IEEE Transactions on Automatic Control, 46 (12), 1941-1946.

ApS, M., 2015. The MOSEK optimization toolbox for MATLAB manual. Version 7.1 (Revision 28).

Arzelier, D., Peaucelle, D., and Salhi, S., 2003. Robust static output feedback stabilization for polytopic uncertain systems: improving the guaranteed performance bound, IFAC Proceedings Volumes, 36 (11), 425-430.

Boukas, E.K. and Shi, P., 1998. Stochastic stability and guaranteed cost control of discrete-time uncertain systems with Markovian jumping parameters, International Journal of Robust and Nonlinear Control, 8 (13), 1155-1167.

Chen, W.H., Guan, Z.H., and Lu, X., 2003. Delay-dependent guaranteed cost control for uncertain discrete-time systems with delay, IEE Proceedings-Control Theory and Applications, 150 (4), 412-416.

Cuzzola, F.A., Geromel, J.C., and Morari, M., 2002. An improved approach for constrained robust model predictive control, Automatica, 38 (7), 1183-1189.

de Oliveira, M.C., Bernussou, J., and Geromel, J.C., 1999a. A new discrete-time robust stability condition, Systems $\&$ control letters, 37 (4), 261-265.

de Oliveira, M.C., Bernussou, J., and Geromel, J.C., 1999b. A new discrete-time robust stability condition, Systems \& control letters, 37 (4), 261-265.

de Souza, C.E., 2003. A mode-independent $H \infty$ filter design for discrete-time Marko- 
vian jump linear systems, in: 42nd Conference on Decision and Control. Proceedings, IEEE, vol. 3, 2811-2816.

de Souza, C.E. and Xie, L., 1992. On the discrete-time bounded real lemma with application in the characterization of static state feedback $H \infty$ controllers, Systems $\&$ Control Letters, 18 (1), 61-71.

Dong, J. and Yang, G.H., 2007. Static output feedback control synthesis for linear systems with time-invariant parametric uncertainties, IEEE Transactions on Automatic Control, 52 (10), 1930-1936.

Donkers, M., 2017. Decentralized robust controller synthesis for discrete-time polytopic systems with additive uncertainty using an iterative-LMI approach, in: American Control Conference (ACC). Proceedings, IEEE, 3096-3101.

Douglas, J.S., 1991. Linear quadratic control for systems with structured uncertainty, Ph.D. thesis, Massachusetts Institute of Technology.

Feron, E., 1997. Analysis of robust $H_{2}$ performance using multiplier theory, SIAM Journal on Control and Optimization, 35 (1), 160-177.

Gahinet, P. and Apkarian, P., 1994. A linear matrix inequality approach to $H \infty$ control, International journal of robust and nonlinear control, 4 (4), 421-448.

Garcia, G., Pradin, B., Tarbouriech, S., and Zeng, F., 2003. Robust stabilization and guaranteed cost control for discrete-time linear systems by static output feedback, Automatica, 39 (9), 1635-1641.

Graham, M., de Oliveira, M., and de Callafon, R., 2006. A linear matrix inequality for robust stability analysis with frequency-dependent multipliers, in: 45th Conference on Decision and Control. Proceedings, IEEE, 5144-5149.

He, Y., Wu, M., She, J.H., and Liu, G.P., 2004. Parameter-dependent Lyapunov functional for stability of time-delay systems with polytopic-type uncertainties, IEEE Transactions on Automatic Control, 49 (5), 828-832.

Iwasaki, T., Meinsma, G., and Fu, M., 2000. Generalized S-procedure and finite frequency KYP lemma, Mathematical Problems in Engineering, 6 (2-3), 305-320.

Kalman, R.E. et al., 1960. Contributions to the theory of optimal control, Bol. Soc. Mat. Mexicana, 5 (2), 102-119.

Köroğlu, H. and Falcone, P., 2014. New LMI conditions for static output feedback synthesis with multiple performance objectives, in: 53rd Conference on Decision and Control. Proceedings, IEEE, 866-871.

Lee, K.H., Lee, J.H., and Kwon, W.H., 2006. Sufficient LMI conditions for $\mathrm{H}_{2} / \mathrm{H}_{\infty}$ output feedback stabilization of linear discrete-time systems, IEEE Transactions on Automatic Control, 51 (4), 675-680.

Li, J., Chen, Z., Cai, D., Zhen, W., and Huang, Q., 2016. Delay-dependent stability control for power system with multiple time-delays, IEEE Transactions on Power Systems, 31 (3), 2316-2326.

Löfberg, J., 2004. Yalmip: A toolbox for modeling and optimization in MATLAB, in: International Symposium on Computer Aided Control Systems Design. Proceedings, IEEE, 284-289.

Lu, J., Li, D., and Xi, Y., 2013. Constrained model predictive control synthesis for uncertain discrete-time Markovian jump linear systems, IET Control Theory $\&$ Applications, 7 (5), 707-719.

Moheimani, S.R. and Petersen, I.R., 1996. Optimal guaranteed cost control of uncertain systems via static and dynamic output feedback, Automatica, 32 (4), 575-579.

Morais, C.F., Braga, M.F., Oliveira, R.C., and Peres, P.L., 2016. Reduced-order dynamic output feedback control of uncertain discrete-time Markov jump linear systems, International Journal of Control, 1-16. 
Moreira, H.R., Oliveira, R.C., and Peres, P.L., 2011. Robust $H_{2}$ static output feedback design starting from a parameter-dependent state feedback controller for timeinvariant discrete-time polytopic systems, Optimal Control Applications and Methods, 32 (1), 1-13.

Packard, A. and Doyle, J., 1993. The complex structured singular value, Automatica, 29 (1), 71-109.

Paganini, F., 2013. Robust $\mathrm{H}_{2}$ Performance in Feedback Control, London: Springer London, 1-10.

Peaucelle, D. and Arzelier, D., 2001. An efficient numerical solution for $\mathrm{H}_{2}$ static output feedback synthesis, in: European Control Conference (ECC). Proceedings, IEEE, 3800-3805.

Peaucelle, D. and Ebihara, Y., 2014. LMI results for robust control design of observerbased controllers, the discrete-time case with polytopic uncertainties, IFAC Proceedings Volumes, 47 (3), 6527-6532.

Petersen, I.R., 1987. A stabilization algorithm for a class of uncertain linear systems, Systems \& Control Letters, 8 (4), 351-357.

Petersen, I.R., McFarlane, D.C., and Rotea, M.A., 1998. Optimal guaranteed cost control of discrete-time uncertain linear systems, International Journal of Robust and Nonlinear Control, 8 (8), 649-657.

Qiu, L., Yao, F., Xu, G., Li, S., and Xu, B., 2016. Output feedback guaranteed cost control for networked control systems with random packet dropouts and time delays in forward and feedback communication links, IEEE Transactions on Automation Science and Engineering, 13 (1), 284-295.

Sadeghzadeh, A., 2014. Fixed-order $\mathrm{H}_{2}$ controller design for state space polytopic systems, International Journal of Control, Automation and Systems, 12 (2), 316323.

Sadeghzadeh, A. and Karimi, A., 2015. Fixed-structure $H_{2}$ controller design for polytopic systems via LMIs, Optimal Control Applications and Methods, 36 (6), 794-809.

Sadeghzadeh, A. and Momeni, H., 2016. Robust output feedback control for discretetime systems with ellipsoidal uncertainty, IMA Journal of Mathematical Control and Information, 33 (4), 911-932.

Safonov, M.G. and Athans, M., 1976. Gain and phase margin for multiloop LQG regulators., Tech. rep., DTIC Document.

Shamma, J.S., 1994. Robust stability with time-varying structured uncertainty, IEEE Transactions on Automatic Control, 39 (4), 714-724.

Shen, M., Ye, D., Fei, S., and Park, J.H., 2015. A new approach to static output control of uncertain continuous Markov jump linear systems, Circuits, Systems, and Signal Processing, 34 (8), 2517-2535.

Sznaier, M., Amishima, T., Parrilo, P.A., and Tierno, J., 2002. A convex approach to robust $\mathrm{H}_{2}$ performance analysis, Automatica, 38 (6), 957-966.

Terra, M.H., Cerri, J.P., and Ishihara, J.Y., 2014. Optimal robust linear quadratic regulator for systems subject to uncertainties, Automatic Control, IEEE Transactions on, 59 (9), 2586-2591.

Wang, L., Wang, Y., Wang, X., and Lian, H., 2016. Robust guaranteed cost faulttolerant control for uncertain networked control system with time delay and packet dropout, in: 28th Chinese Control and Decision Conference (CCDC). Proceedings, IEEE, 2607-2612.

Wu, F. and Lu, B., 2004. On convexified robust control synthesis, Automatica, 40 (6), $1003-1010$.

Xia, Y., Liu, G.P., Shi, P., Chen, J., and Rees, D., 2008. Robust constrained model 
predictive control based on parameter-dependent Lyapunov functions, Circuits, Systems 83 Signal Processing, 27 (4), 429-446.

Xie, L., 1996. Output feedback $H \infty$ control of systems with parameter uncertainty, International Journal of control, 63 (4), 741-750.

Xie, L. and Soh, Y.C., 1993. Control of uncertain discrete-time systems with guaranteed cost, in: 32th Conference on Decision and Control. Proceedings, IEEE, 56-61.

Yang, D. and Zhao, J., 2016. Guaranteed cost control for LPV systems with Markovian switching under partially known transition rates, in: 35th Chinese Control Conference (CCC). Proceedings, IEEE, 5774-5779.

$\mathrm{Yu}, \mathrm{L}$. and Chu, J., 1999. An LMI approach to guaranteed cost control of linear uncertain time-delay systems, Automatica, 35 (6), 1155-1159.

\section{Appendix A. Proof of Lemma 4.3 for the Optimal Guaranteed Cost Control}

In this appendix, we prove Lemma 4.3 . Towards this goal, we first present supporting theory required for the proof.

Lemma A.1. For all $i \in[1, n]$, let $\mathbb{S}_{i}$ be lossless sets. Then,

$$
\mathbb{H}\left(\operatorname{conv}\left(\bigcup_{i=1}^{n} \mathbb{S}_{i}\right)\right)=\mathbb{H}\left(\bigcup_{i=1}^{n} \mathbb{S}_{i}\right)=\bigcap_{i=1}^{n} \mathbb{H}\left(\mathbb{S}_{i}\right)
$$

where $\mathbb{H}(\mathbb{S})$ is the set of matrices $H$ that satisfy property (iii) of Definition 3.1.

Proof. Due to the associative property of the involved operations, we will restrict the prove, without loss of generality, to the case $n=2$. We will first prove the second equality, then proceed to prove the first.

Let $H \in \mathbb{H}\left(\mathbb{S}_{0}\right) \cap \mathbb{H}\left(\mathbb{S}_{1}\right)$. Then,

$$
\forall S \in \mathbb{S}_{0} \cup \mathbb{S}_{1}: \operatorname{tr}(S H) \leq 0
$$

and

$$
H=\sum_{i=0}^{\operatorname{rank}(H)} \lambda_{i} \lambda_{i}^{*}, \forall S \in \mathbb{S}_{0} \cup \mathbb{S}_{1}: \lambda_{i}^{*} S \lambda_{i} \leq 0
$$

Therefore, $H \in \mathbb{H}\left(\mathbb{S}_{0} \cup \mathbb{S}_{1}\right)$ and $\mathbb{H}\left(\mathbb{S}_{0} \cup \mathbb{S}_{1}\right)=\mathbb{H}\left(\mathbb{S}_{0}\right) \cap \mathbb{H}\left(\mathbb{S}_{1}\right)$.

Consider now the following set:

$$
\mathbb{S}_{2}=\operatorname{conv}\left(\mathbb{S}_{0} \cup \mathbb{S}_{1}\right)=\left\{\alpha_{0} S_{0}+\alpha_{1} S_{1} \mid S_{0} \in \mathbb{S}_{0}, S_{1} \in \mathbb{S}_{1}, \alpha_{0} \geq 0, \alpha_{1} \geq 0\right\},
$$

and $H \in \mathbb{H}\left(\mathbb{S}_{2}\right)$. Then, from property (iii), we obtain

$$
\forall \alpha_{0} \geq 0, \alpha_{1} \geq 0, S_{0} \in \mathbb{S}_{0}, S_{1} \in \mathbb{S}_{1}, \alpha_{0} \operatorname{tr}\left(S_{0} H\right)+\alpha_{1} \operatorname{tr}\left(S_{1} H\right) \leq 0
$$


and

$$
H=\sum_{i=0}^{\operatorname{rank}(H)} \lambda_{i} \lambda_{i}^{*}, \alpha_{0} \lambda_{i}^{*} S_{0} \lambda_{i}+\alpha_{1} \lambda_{i}^{*} S_{1} \lambda_{i} \leq 0 .
$$

If $H$ is chosen such that $H \in \mathbb{H}\left(\mathbb{S}_{0} \cup \mathbb{S}_{1}\right)$, then $\left.\mathrm{A} 5\right)$ and $(\mathrm{A} 6)$ are immediately satisfied. However, if we choose $H$ such that $H \notin \mathbb{H}\left(\mathbb{S}_{0} \cup \mathbb{S}_{1}\right)$, it is impossible to satisfy A5 and $(\mathrm{A} 6)$ for cases $\alpha_{0}=1, \alpha_{1}=0$ and $\alpha_{0}=0, \alpha_{1}=1$. Therefore, $\mathbb{H}\left(\operatorname{Conv}\left(\mathbb{S}_{0} \cup \mathbb{S}_{1}\right)\right)=$ $\mathbb{H}\left(\mathbb{S}_{0} \cup \mathbb{S}_{1}\right)$.

Corollary A.2. The convex hull of unions of lossless sets is also lossless, since it is a convex cone (properties (i) and (ii) hold) and property (iii) holds from Lemma A.1.

Lemma A.3. Let $C \in \Re^{r(p+q) \times n}$, and $Q \in \Re^{r \times r}$ be a positive semi-definite matrix. Then, the set

$$
\mathbb{S}=\left\{C^{T}\left[\begin{array}{cc}
Q \otimes I_{p} & 0 \\
0 & -Q \otimes I_{q}
\end{array}\right] C \mid \forall Q \succ 0\right\}
$$

is lossless.

Proof. (Based on proof of Lemma 3 of (Iwasaki et al. 2000)) Properties (i) and (ii) from Definition 3.1 are trivial. Therefore, we focus on proving property (iii) holds.

Let

$$
S=C^{T}\left[\begin{array}{cc}
Q \otimes I_{p} & 0 \\
0 & -Q \otimes I_{q}
\end{array}\right] C
$$

for brevity. Then, from the properties of Kronecker product, there exists a self-similar transformation $T$ such that

$$
S=C^{T} T^{T}\left[\begin{array}{cc}
I_{p} \otimes Q & 0 \\
0 & -I_{q} \otimes Q
\end{array}\right] T C
$$

Let $H \in \Re^{n \times n}$ be a positive semi-definite matrix such that $\operatorname{tr}(H S) \leq 0$ for all $S \in \mathbb{S}$. Then, $H$ admits a full rank factorization $H=G G^{T}$ where $G \in \Re^{n \times r}$ where $r=\operatorname{rank}(H)$. We define

$$
\left[\begin{array}{c}
W \\
Z
\end{array}\right]=T C G
$$




$$
\begin{aligned}
W=\left[W_{1}^{T}, W_{2}^{T}, \ldots, W_{p}^{T}\right]^{T}, & \text { and } Z=\left[Z_{1}^{T}, Z_{2}^{T}, \ldots, Z_{q}^{T}\right]^{T} \text {. Then }, \\
\operatorname{tr}(H S) & =\operatorname{tr}\left(G G^{T} S\right)=\operatorname{tr}\left(G^{T} S G\right) \\
& =\operatorname{tr}\left(\left[\begin{array}{c}
W \\
Z
\end{array}\right]^{T}\left[\begin{array}{cc}
I_{p} \otimes Q & 0 \\
0 & -I_{q} \otimes Q
\end{array}\right]\left[\begin{array}{c}
W \\
Z
\end{array}\right]\right) \\
& =\operatorname{tr}\left(\sum_{i=1}^{p} W_{i}^{T} Q W_{i}-\sum_{i=1}^{q} Z_{i}^{T} Q Z_{i}\right) \\
& =\sum_{i=1}^{p} \operatorname{tr}\left(W_{i}^{T} Q W_{i}\right)-\sum_{i=1}^{q} \operatorname{tr}\left(Z_{i}^{T} Q Z_{i}\right) \\
& =\sum_{i=1}^{p} \operatorname{tr}\left(W_{i} W_{i}^{T} Q\right)-\sum_{i=1}^{q} \operatorname{tr}\left(Z_{i} Z_{i}^{T} Q\right) \leq 0 .
\end{aligned}
$$

Notice that A11 must hold for all $Q \succeq 0$. Then, since $W^{T} W=\sum_{i=1}^{p} W_{i}^{T} W_{i}$ and $Z^{T} Z=\sum_{i=1}^{p} Z_{i}^{T} Z_{i}, \mathrm{~A} 11$ is equivalent to

$$
W^{T} W-Z^{T} Z \preceq 0
$$

which also implies that $W^{T}\left(I_{p} \otimes Q\right) W-Z^{T}\left(I_{q} \otimes Q\right) Z \preceq 0$, since $I \otimes Q \succeq 0$.

Assume that (iii) doesn't hold, then there exists $\xi_{i} \in \Re^{n}$ such that $H=\sum_{i=1}^{r} \xi_{i} \xi_{i}^{T}$, $\operatorname{tr}(H S) \leq 0 \forall S \in \mathbb{S}$, and $\xi_{i}^{T} S \xi_{i}>0$ for some $i$ and $S \in \mathbb{S}$.

Let $\xi_{i}=G u_{i}$ where $u_{i} \in \Re^{r},\left\|u_{i}\right\|_{2}=1$ and $\sum_{i=1}^{r} u_{i} u_{i}^{T}=I$. Then,

$$
\xi_{i}^{T} S \xi_{i}=u_{i}^{T} G^{T} S G u_{i}=u_{i}^{T}\left(W^{T}\left(I_{p} \otimes Q\right) W-Z^{T}\left(I_{q} \otimes Q\right) Z\right) u_{i}>0
$$

and $\operatorname{tr}(H S)>0$, which contradicts the initial assumption. Therefore, (iii) holds and $\mathbb{S}$ is lossless.

We are now ready to prove Lemma 4.3 .

Proof. We first prove property (i) $\left(\mathbb{S}^{u} \subseteq \mathbb{S}\right.$ ), then continue to prove property (ii) ( $\mathbb{S}$ is lossless).

(i): Let $\Lambda_{p}=\tau I_{n_{p}}$ and $\Lambda_{q}=\tau I_{n_{q}}$. Then, $\mathbb{S}$ reduces to $\mathbb{S}^{u}$. Therefore, $\mathbb{S}^{u} \subseteq \mathbb{S}$.

(ii): For all $i \in[1, s]$, let $\mathbb{S}_{i}=\left\{S_{i}^{s}\left(\Lambda_{i}\right) \mid \forall \Lambda_{i} \succeq 0\right\}$, where

$$
S_{i}^{s}\left(\Lambda_{i}\right)=\left[\begin{array}{cc}
\bar{C}_{z, i} & \bar{D}_{z, i}^{w} \\
0 & I_{n_{r i} n_{q i}}
\end{array}\right]^{T}\left[\begin{array}{cc}
\Lambda_{i} \otimes I_{n_{p i}} & 0 \\
0 & -\Lambda_{i} \otimes I_{n_{q i}}
\end{array}\right](\bullet) .
$$

Then, we conclude that

$$
\mathbb{S}=\operatorname{conv}\left(\bigcup_{i=1}^{s} \mathbb{S}_{i}\right)
$$


Based on Lemma A.3 and Corollary A.2, $S_{i}$ is lossless for all $i \in[1, s]$ and $\mathrm{S}$ is lossless since it is the convex hull of the union of lossless sets. 